\title{
Gene ontology analysis of expanded porcine blastocysts from gilts fed organic or inorganic selenium combined with pyridoxine
}

\author{
Danyel Bueno Dalto ${ }^{1 *}$, Stephen Tsoi ${ }^{2}$, Michael K. Dyck ${ }^{2}$ and Jean-Jacques Matte ${ }^{1}$
}

\begin{abstract}
Background: Gene ontology analysis using the microarray database generated in a previous study by this laboratory was used to further evaluate how maternal dietary supplementation with pyridoxine combined with different sources of selenium (Se) affected global gene expression of expanded porcine blastocysts. Data were generated from 18 gilts randomly assigned to one of three experimental diets ( $n=6$ per treatment): i) basal diet without supplemental Se or pyridoxine (CONT); ii) CONT $+0.3 \mathrm{mg} / \mathrm{kg}$ of Na-selenite and $10 \mathrm{mg} / \mathrm{kg}$ of $\mathrm{HCl}$-pyridoxine $\left(\mathrm{MSeB}_{6} 10\right)$; and iii) CONT $+0.3 \mathrm{mg} / \mathrm{kg}$ of Se-enriched yeast and $10 \mathrm{mg} / \mathrm{kg}$ of $\mathrm{HCl}$-pyridoxine $\left(\mathrm{OS}_{6} \mathrm{~B}_{6} 10\right)$. All gilts were inseminated at their fifth post-pubertal estrus and euthanized 5 days later for embryo harvesting. Differential gene expression between $\mathrm{MSeB}_{6} 10$ vs CONT, OSeB 10 vs CONT and $\mathrm{OSeB}_{6} 10$ vs MSeB 10 was performed using a porcine embryo-specific microarray.

Results: There were 559, 2458, and 1547 differentially expressed genes for MSeB 10 vs CONT, OSeB 10 vs CONT and $\mathrm{OSeB}_{6} 10$ vs $\mathrm{MSeB}_{6} 10$, respectively. $\mathrm{MSeB}_{6} 10$ vs CONT stimulated 13 biological processes with a strict effect on RNA binding and translation initiation. $\mathrm{OSeB}_{6} 10$ vs CONT and $\mathrm{OSeB}_{6} 10$ vs MSeB 10 impacted 188 and 66 biological processes, respectively, with very similar effects on genome stability, ceramide biosynthesis, protein trafficking and epigenetic events. The stimulation of genes related with these processes was confirmed by quantitative real-time RT-PCR.
\end{abstract}

Conclusions: Gene expression of embryos from $\mathrm{OSeB}_{6} 10$ supplemented gilts was more impacted than those from $\mathrm{MSeB}_{6} 10$ supplemented gilts. Whereas maternal $\mathrm{OSeB}_{6} 10$ supplementation influenced crucial aspects of embryo development, maternal $\mathrm{MSe}_{6} 10$ supplementation was restricted to binding activity.

Keywords: Amide biosynthesis, Epigenetic, Genomic stability, Peptide trafficking, Pyridoxine, Porcine embryo, Selenium

\section{Background}

Although selenium (Se) acts as part of hormones and enzymes influencing the activity of all organs and tissues, the major metabolic role of Se in the body is related to selenoproteins and the antioxidant system. However, in embryos, fetuses, and newborns, the synthesis of selenoproteins from Se-methionine (SeMet) is impaired due to the

\footnotetext{
* Correspondence: danyel.buenodalto@canada.ca

${ }^{1}$ Sherbrooke Research and Development Centre, Agriculture and Agri-Food

Canada, 2000 College Street, Sherbrooke, QC J1M 0C8, Canada

Full list of author information is available at the end of the article
}

inactivity of cystathionine gamma lyase in the metabolic pathway, in spite of its mRNA expression [1, 2]. This would imply that, from conception to neonatal age, individuals are not able to convert SeMet into Se-cysteine (SeCys) via the pyridoxine $\left(\mathrm{B}_{6}\right)$-dependent transsulfuration pathway. In pre-implantation porcine embryos, Dalto et al. [3] showed that, although significant differences in genes expression were observed between embryos from dams supplemented with dietary organic (OSe) vs inorganic Se (MSe), the uterine flushing is a negligible source of Se.

(C) The Author(s). 2018 Open Access This article is distributed under the terms of the Creative Commons Attribution 4.0 International License (http://creativecommons.org/licenses/by/4.0/), which permits unrestricted use, distribution, and 
These two crucial aspects suggest that, in pre-implantation embryos, SeMet has an alternative metabolism not related with the antioxidant system and that other sources of Se are transferred from the dam in order to allow selenoprotein synthesis. Because of its undiscriminated metabolism in relation to methionine [4], SeMet is most likely incorporated into general protein, excreted through methylation reactions or recycled after demethylation to Se-homocysteine, generating the Se-derived adenosylmethionine [5]. S-adenosylmethionine (SAM), the sulfur counterpart of Se-adenosylmethionine, is recognized as universal bioactive methyl donor donating its methyl group to a large number of methyl acceptors $[6,7]$ with profound impacts on DNA synthesis, DNA protection and repair, cellular metabolism, and cell proliferation. Consequently, it can generate direct effects on embryo/ fetal growth [8,9] and epigenetic modifications [10]. Regarding the transfer of Se from dam to pre-implantation embryos, to the best of our knowledge, this aspect of SeMet metabolism is not known in porcine embryos developing under such specific uterus conditions. In a previous study using a unique microarray platform, Dalto et al. [3] have evaluated genes expression of porcine expanded blastocysts (PEB) from gilts fed organic or inorganic Se and $\mathrm{B}_{6}$. In that report, the focus was put on aspects related with Se metabolism and antioxidation, using a very restrictive gene selection approach. Therefore, potential global metabolic effects of Se on PEB were not addressed.

Considering the uniqueness of these samples (embryos collected at day 5 of gestation from gilts under specific dietary treatments and controlled experimental conditions), complementary analyses were performed on samples and on the dataset from the previous study. In the present report, a gene ontology approach was applied to provide a global overview of the effects of Se in PEB and, considering the lack of information on that matter, these new findings allowed proposing hypotheses in relation to Se metabolism at this stage of development.

\section{Material and methods}

Most aspects of the Materials and Methods for the present study have been previously described in Dalto et al. [3]. Therefore, the present description summarizes only the relevant information needed for the present objectives.

Eighteen Yorkshire-Landrace gilts were selected at $96.1 \pm 4.6 \mathrm{~kg}$ of body weight and 135-170 days of age. For at least 14 days, they were fed ad libitum a basal breeding/gestation diet without $\mathrm{Se}$ and $\mathrm{B}_{6}$ supplements but in excess of the recommended NRC [11] requirements for all other ingredients. Estrus detection was initially performed once daily, but was increased to twice daily for the detection of the fifth post-pubertal estrus. From the onset of the first post-pubertal estrus, gilts were placed in individual stalls, daily feed allowance was limited to $2.8 \mathrm{~kg}$, and they were randomly assigned (according to their body weight and blood concentration of $\mathrm{Se})$ to one of the three experimental diets $(n=6$ per treatment): i) basal diet containing 0.3 and $2.4 \mathrm{mg} / \mathrm{kg}$ of native Se and pyridoxine, respectively (CONT); ii) basal diet + 0.3 and $10 \mathrm{mg} / \mathrm{kg}$ of supplemental inorganic Se (Na-selenite) and $\mathrm{HCl}$-pyridoxine, respectively $\left(\mathrm{MSeB}_{6} 10\right)$; and iii) basal diet +0.3 and $10 \mathrm{mg} / \mathrm{kg}$ of supplemental organic Se (Se-enriched yeast) and $\mathrm{HCl}$-pyridoxine, respectively $\left(\mathrm{OSeB}_{6} 10\right)$. At the fifth post-pubetal estrus, all gilts were inseminated with pooled semen from the same three Duroc boars. When estrus was detected in the morning, gilts were inseminated 8 and $24 \mathrm{~h}$ later. When estrus was detected in the afternoon, inseminations were performed 16 and $24 \mathrm{~h}$ later. All gilts were euthanized 5 days after the first insemination. The euthanasia procedure involved the sedation of the animal (Stresnil $2.5 \mathrm{ml} / \mathrm{kg}$; Vetoquinol) followed by stunning using a penetrating captive bolt (TED; Bock Industries) and bleeding. Average body weight was $138.5 \pm 6.3$ and $181.3 \pm 6.3 \mathrm{~kg}$ at the initiation of treatment and at the end of the experiment, respectively.

All procedures related with embryo (day 5 of gestation) collection and storage, total RNA extraction, microarray processes, and bioinformatics tools were previously described by Dalto et al. [3]. A two-color microarray with a dye-swap replicate was performed for the $\mathrm{MSeB}_{6} 10$ vs CONT and $\mathrm{OSeB}_{6} 10$ vs CONT comparisons and a reference design [12] was chosen using the same CONT group used in the previous comparisons as a reference for a reliable indirect comparison of gene expression for $\mathrm{OSeB}_{6} 10$ vs $\mathrm{MSeB}_{6} 10$. Annotation of all the unknown genes was performed using porcine Sscrofa10.2 database (accession GCF_000003025.5). The list of 14,536 unique gene symbols (GSs) from re-annotated EMPV1 used as background for Gene Ontology (GO) Enrichment Analysis is shown in the Additional file 1: Table S1. GORILLA Classification System (http://cbl-gorilla.cs.technion.ac.il/) was used for the GO analysis with two unranked lists of genes (target and background lists). These results were further uploaded to REVIGO (http://revigo.irb.hr/) to provide a summarized view of results.

\section{Quantitative real-time RT-PCR (RT-qPCR) and analysis}

A two-step quantitative real-time RT-PCR (RT-qPCR) was performed on the same aRNA samples from Dalto et al. [3]. Based on the microarray data, two genes related with epigenetic events: histone acetyltransferase 1 (HAT1) and histone deacetylase 1 (HDAC1); two genes related with establishment of protein localization to endoplasmic reticulum: signal peptidase complex subunit 1 (SPCS1) and signal recognition particle 9 (SRP9); two genes related with protein catabolism: anaphase promoting complex subunit 1 (ANAPC1) and cullin 1 (CUL1); one gene 
related with transcription initiation and DNA repair: cyclin dependent kinase 7 (CDK7); and one gene related with the nucleotide excision repair system: growth arrest and DNA-damage-inducible gamma interacting protein 1 (GADD45GIP1) were chosen for RT-qPCR validation using the methodology described by Dalto et al. [3]. The reference gene that was found to be the least affected by treatments and, therefore, used for normalization was peptidylprolyl isomerase A (PPIA). The sequence information of the primers is given in Table 1.

For RT-qPCR analysis, the Relative Expression Software Tool 2009 (REST; http://rest.gene-quantification.info/) was used to implement a randomized test [13] and to assess statistical significance of the up- or down-regulation of the target genes after normalization to the reference gene. Statistical analyses were considered significant at $P \leq 0.05$.

\section{Results}

\section{Differentially genes expression profiles}

The direct comparison of $\mathrm{MSeB}_{6} 10$ vs CONT embryos showed a total of 559 genes in the PEB, with 293 up-regulated (52.4\%) and 266 down-regulated (47.6\%) in $\mathrm{MSeB}_{6} 10$. For the $\mathrm{OSeB}_{6} 10$ vs CONT comparison, a total of 2458 differentially expressed genes were found, with 1658 up-regulated (67.5\%) and 800 down-regulated genes $(32.5 \%)$ in $\mathrm{OSeB}_{6} 10$. Regarding the reference design comparison $\left(\mathrm{OSeB}_{6} 10\right.$ vs $\left.\mathrm{MSeB}_{6} 10\right)$, there were 1547 genes from which $\mathrm{OSeB}_{6} 10$ had 1096 up-regulated (70.8\%) and 451 down-regulated genes (29.2\%) compared with $\mathrm{MSeB}_{6} 10$. Detailed information on the above comparison between treatments (EMPV1 probe ID, log2 fold change, $P$-value, and gene symbol) are given in Additional file 2: Table S2.

\section{Gene ontology enrichment analyses}

Regarding the $\mathrm{MSeB}_{6} 10$ vs CONT comparison, 6 distinct cellular components, 1 molecular function, and 13 biological processes were stimulated $\left(P \leq 8.94 \times 10^{-8}\right.$; FDR q-value $\leq 9.57 \times 10^{-5}$ ) according to GORILLA, which were respectively included in 2, 1 and 5 GO terms on REVIGO $\left(\log _{10} P \leq-7.05\right)$. The summarized description of REVIGO shows that $\mathrm{MSeB}_{6} 10$ stimulated cellular components of the cytosol, more specifically intracellular organelle parts and the macromolecular complex, functioning as a RNA binding element. The metabolic role of $\mathrm{MSeB}_{6} 10$ in PEB is related with cellular metabolism, especially nucleobase-containing compound metabolic process. Although not categorized as cellular metabolism, other GO term identified was translational initiation. By analyzing each individual gene of the $\mathrm{MSeB}_{6} 10$ $x$ CONT dataset, few genes related with epigenetic events were found [HDAC1, HDAC9, lysine demethylase $5 \mathrm{C}($ KDM5C) and 5 methyltransferases].

For the comparison of $\mathrm{OSeB}_{6} 10$ vs CONT, 47, 20, and 118 cellular components, molecular functions, and biological processes were respectively stimulated $(P \leq 8.30 \times$ $10^{-8}$; FDR q-value $\leq 1.15 \times 10^{-5}$ ) according to GORILLA, which were included in $5,12,28 \mathrm{GO}$ terms on REVIGO respectively $\left(\log _{10} P \leq-7.01\right)$. The summarized description of REVIGO shows that $\mathrm{OSeB}_{6} 10$ stimulated cellular components of the nucleus and catalytic complex. A more detailed observation reveals that $\mathrm{OSeB}_{6} 10$ is widely distributed within the cell, being a component of the nucleus

Table 1 Primer sequences used for RT-qPCR amplifications of reference gene and selected genes in porcine expanded blastocysts

\begin{tabular}{|c|c|c|c|c|}
\hline Genes & Primer sequences $\left(5^{\prime} \rightarrow 3^{\prime}\right)$ & GenBank Accession no. & Product size (bp) & Amplification efficiency (\%) \\
\hline \multirow[t]{2}{*}{ CDK7 } & (F) TGGAATCCCGCTACAACATATC & XM_021076538.1 & 111 & 0.997 \\
\hline & (R) AATGCCTGTGTGGCTGTAA & & & \\
\hline \multirow[t]{2}{*}{ SPCS1 } & (F) TGGATTACAAGGGCCAGAAG & NM_001114288.2 & 91 & 0.996 \\
\hline & (R) GCCACGTACCCGTAGATAAAT & & & \\
\hline \multirow[t]{2}{*}{ GADD45GIP1 } & (F) ATTGAAGAGTGCATGGCTAAGA & XM_003123339.3 & 89 & 0.999 \\
\hline & (R) TTGTCTGCTTGCTCCTTCTC & & & \\
\hline \multirow[t]{2}{*}{ ANAPC1 } & (F) CCTGTTTCCTTGTCTACCACTC & XM_013995810.1 & 118 & 0.999 \\
\hline & (R) ACTGGCATCTTGAGCTGTTTA & & & \\
\hline \multirow[t]{2}{*}{ HDAC1 } & (F) GGGATTGATGACGAGTCCTATG & XM_013999116.2 & 104 & 0.999 \\
\hline & (R) GAGTCAGAGCCACACTGTAAG & & & \\
\hline \multirow[t]{2}{*}{ CUL1 } & (F) CAGTTACTCGGAGAAGTCCTAAC & XM_013979868.1 & 117 & 0.999 \\
\hline & (R) ACCATCAACTCGCTCCAAATA & & & \\
\hline \multirow[t]{2}{*}{ SRP9 } & (F) CCTGCCCAATTCTCCCTTTAT & XM_003130540.6 & 90 & 0.994 \\
\hline & (R) CAATCCCATACTTCCGGTTTACT & & & \\
\hline \multirow[t]{2}{*}{ HAT1 } & (F) GCAGTAGAGGCTCAACAGAAG & XM_003483674.4 & 91 & 0.999 \\
\hline & (R) CACTCATGTCAGTTACCAGTAGTC & & & \\
\hline
\end{tabular}


(spliceosome complex), mitochondria (matrix and inner membrane protein complex), and ribosomes as well as ribonucleoprotein complex (related with both ribosome and spliceosomal complex), and oxidoreductase complex. According to REVIGO, the functions of $\mathrm{OSeB}_{6} 10$ are mainly related with protein binding and translation initiation factor activity. Among these two metabolic functions the most representative GO terms were protein binding and translation factor activity - RNA binding. Although not related with those two main metabolic functions, other interesting GO terms were NADH dehydrogenase (ubiquinone) activity, structural constituent of ribosome, and ribonucleoprotein complex binding. Among the 28 biological processes on REVIGO for $\mathrm{OSeB}_{6} 10 \times \mathrm{CONT}$, results show that RNA processing, establishment of protein localization to organelle, and macromolecular complex subunit organization are the most relevant. Among these processes, GO terms related with DNA repair, mRNA processing, mRNA translation, intracellular ceramides synthesis, and intracellular peptides trafficking stood out (Additional file 3: Table S3). By analyzing each individual gene of the $\mathrm{OSeB}_{6} 10 \times \mathrm{CONT}$ dataset, many genes related with epigenetic events were found, among them the most relevant are methyl-CpG binding domain proteins (MBD3, MBD4 and MBD3L5), HAT1, HDAC1, HDAC9, $K D M 5 C$, protein arginine methyltransferases (PRMT7 and PRMT10), DNA methyltransferases (DNMT1 and DNMT3B), and 17 other methyltransferases.

The indirect comparison of Se sources $\left(\mathrm{OSeB}_{6} 10\right.$ vs $\mathrm{MSeB}_{6} 10$ ) showed that 31 cellular components, 10 molecular functions, and 66 biological processes were stimulated $\left(P \leq 9.41 \times 10^{-8}\right.$; FDR q-value $\left.\leq 3.36 \times 10^{-5}\right)$ according to GORILLA and were respectively represented mainly by 4 , 5, 9 GO terms on REVIGO $\left(\log _{10} P \leq-7.34\right)$. The summarized description of REVIGO shows a stimulus in cellular components of the nucleus and catalytic complex. A more detailed observation reveals that Se is widely distributed within the cell, being a component of the nucleus, mitochondria (inner membrane protein complex), and ribosomes. In terms of molecular functions, Se acts mainly in translation factor activity (related with RNA binding). Besides that, Se also acts on protein binding and as a structural constituent of ribosome. Among all biological processes, REVIGO results show that RNA processing and protein localization to endoplasmic reticulum are the most relevant (Additional file 3: Table S3). By analyzing each individual gene of the $\mathrm{OSeB}_{6} 10 \times \mathrm{MseB}_{6} 10$ dataset, many genes related with epigenetic events were found, most of them were similar to those found on $\mathrm{OSeB}_{6} 10 \times \mathrm{CONT}$. In this sense, out of the GORILLA GO terms found in $\mathrm{OSeB}_{6} 10$ vs $\mathrm{MSeB}_{6} 10$ for cellular components, molecular functions, and biological processes, 30 (96.8\%), 10 (100.0\%), and $64(97.0 \%)$, respectively, were also found in the $\mathrm{OSeB}_{6} 10$ vs CONT comparison, indicating that most of the effects observed on $\mathrm{OSeB}_{6} 10$ vs $\mathrm{MSeB}_{6} 10$ came from OSe.

The complete list of GO terms related with each comparison is shown in Additional file 3: Table S3.

\section{Validation of microarray data by RT-qPCR}

Treatment effects on the expression of genes related with the main GO terms were validated by RT-qPCR. The expression of genes CUL1, ANAPC1, SRP9 and $C D K 7$ was up-regulated whereas GADD45GIP1 and SPCS1 were down-regulated in the comparison $\mathrm{OSeB}_{6} 10$ vs CONT (Fig. 1). For $\mathrm{OSeB}_{6} 10$ vs $\mathrm{MSeB}_{6} 10$, RT-qPCR analysis indicated no difference in relative $H A T 1$ expression whereas $H D A C 1$ was up-regulated by a mean factor of 1.88. Selected genes from $\mathrm{MSeB}_{6} 10$ vs CONT comparison did not provide reliable results and were not considered in the present study.

\section{Discussion}

The present study describes a global metabolic perspective of the effects of different Se sources associated with $\mathrm{B}_{6}$ on PEB after maternal supplementation, and generates some hypothesis based on these new findings. Although the same microarray database was used by Dalto et al. [3] and the present study, those authors used the pig genome map draft 9.0 for genes annotation and a more restrictive statistical approach in order to evaluate highly affected processes, functions, and components related with each treatment. In the present study re-annotation was made using the updated pig genome map version 10.2 and parameters related with statistics and genes selection were chosen in order to provide a global overview of the effects of Se in PEB.

Considering that the present results were observed in self-regulated organisms (expanded blastocysts) that are genetically dissimilar (half allogenic) from those directly receiving the treatments (dams), such effects would require major metabolic alterations. In fact, Dalto et al. (2015; original database) reported that maternal blood Se was clearly modulated by dietary Se. Therefore, non-Se fractions of dietary OSe (mainly yeast) or MSe $(\mathrm{Na}+$ ion $)$ are unlikely involved in such major systemic metabolic changes.

Both sources of Se (MSe and OSe) are used as dietary supplements for pigs but, depending on the Se status of the animal, they follow different metabolic pathways [4] before producing a common metabolite (selenide) required for the synthesis of selenoenzymes [5]. According to Burk et al. [14], in mouse, MSe does not take part of the maternal methionine pool but is mainly incorporated in selenoproteins whereas OSe is mostly deposited in the methionine pool with limited synthesis of selenoproteins. Considering that the uterine flushing of 5 days gestation gilts is a negligible route of Se transfer for the 


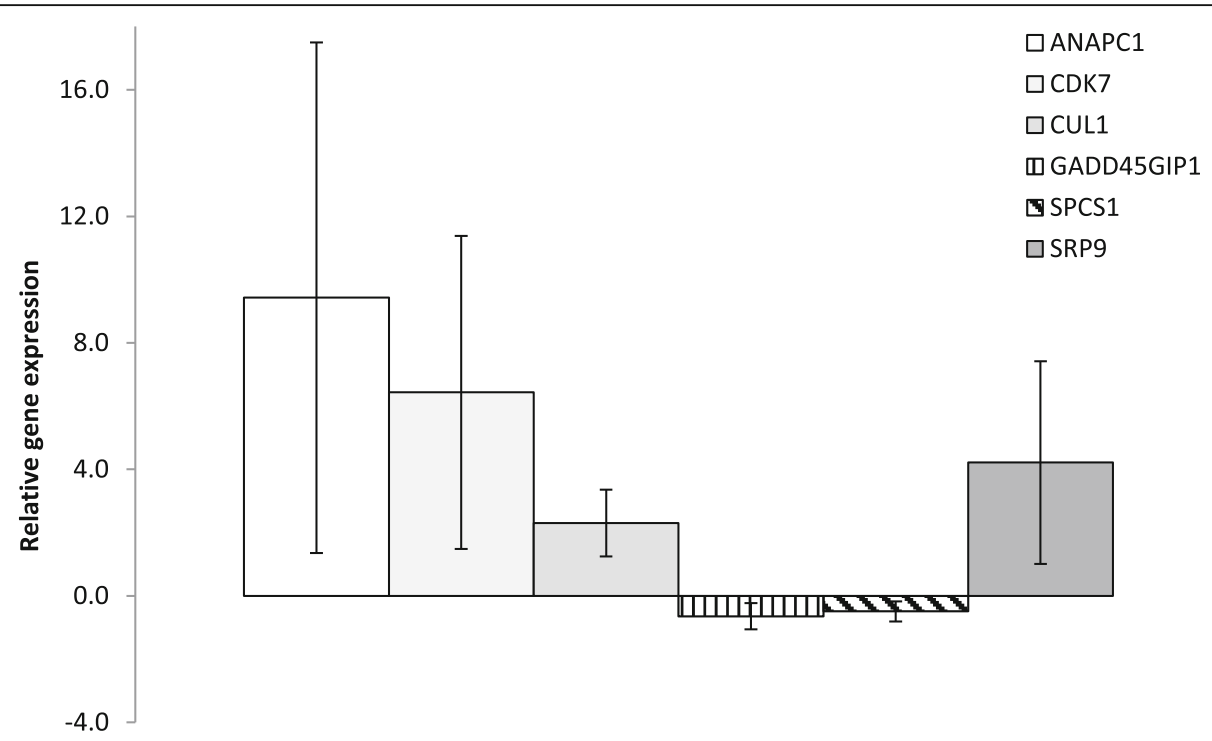

Fig. 1 RT-qPCR expression trends for ANAPC1, CDK7, CUL1, GADD45GIP1, SPCS1, and SRP9 in porcine expanded blastocysts recovered from OSeB 10 supplemented gilts, shown as relative gene expression to CONT ( \pm SEM). PPIA was used to normalize the mRNA expression levels. OSeB $10=$ basal diet supplemented with $0.3 \mathrm{mg} / \mathrm{kg}$ of Se-enriched yeast and $10 \mathrm{mg} / \mathrm{kg}$ of hydro-chloride pyridoxine

embryo [3], the Se content of pre-ovulatory oocytes is likely to be the main source of this mineral for pre-implantation porcine embryos. Therefore, whatever the source of maternal dietary Se, the embryo Se content would derive from an organic metabolite, either the oocyte SeMet pool and/or oocyte selenoproteins.

In this sense, we hypothesize that for both MSe and OSe supplemented gilts, maternal (oocyte) selenoproteins serve, after proteolysis, as a common source of SeCys for either embryo protein deposition or selenoproteins synthesis, whereas oocyte SeMet (OSe) might be directly deposited into embryo proteins and/or undergo transmethylation, because the transsulfuration pathway is not fully functional in embryos [1].

This hypothesis involving a common source of embryonic Se between OSe and MSe is indirectly supported by the present and previous studies. In fact, all $\mathrm{MSeB}_{6} 10$ vs CONT GO terms were also found in $\mathrm{OSeB}_{6} 10$ vs CONT comparison, suggesting that both sources of Se share a common metabolism but also that OSe may have additional metabolic pathways. Fortier et al. [15] and Dalto et al. [16] reported that even after placentation (30 days of gestation) porcine embryos from MSe or OSe supplemented gilts have similar Se-dependent glutathione peroxidase (Se-GPX) activities, indicating that independently of maternal Se source, embryos may obtain their Se content from a common intermediary Se metabolite, which is subjected to similar regulations for the synthesis of Se-GPX. Nevertheless, in both studies, the Se content of embryo from OSe supplemented gilts was greater than in those from MSe supplemented gilts, indicating the presence of different and/or additional Se metabolic pathways in OSe embryos.

\section{DNA repair}

DNA damage triggers repair pathways, but also signalling pathways that stimulate cell cycle checkpoints, apoptosis, transcription, and chromatin remodelling [17]. It has been proposed that, among the mechanisms for monitoring DNA integrity, cells may detect stalled RNA polymerases or abortive transcripts, activating DNA damage signaling [18]. The present results for $\mathrm{OSeB}_{6} 10 \times \mathrm{MSeB}_{6} 10$ indicate that Se acts in one of these signaling pathways, the nucleotide excision repair (NER). More specifically, it affects the transcription-coupled NER (TC-NER), a specialized sub-pathway of NER responsible to repair DNA lesions in transcribed strands and coupled to active transcription $[19,20]$. The high similarity between $\mathrm{GO}$ terms for $\mathrm{OSeB}_{6} 10$ vs $\mathrm{CONT}$ and $\mathrm{OSeB}_{6} 10$ vs $\mathrm{MSeB}_{6} 10$ and the up-regulation of $C D K 7$ (gene that links transcription initiation, DNA repair, and cell cycle) in $\mathrm{OSeB}_{6} 10$ vs CONT suggests a major influence of OSe in this process.

SeMet (main OSe form) have been reported to impact protein p53 activity [21], a protein that under conditions of permanent blockage of transcription may stimulate p53-dependent apoptosis [22]. Therefore, the fast removal of transcription blocking lesions is crucial to avoid the detrimental effects of transcription inhibition. Studies have proposed a direct role of p53 in the OSe-induced activation of the NER pathway through its interaction with NER-associated proteins [23-25]. However, in the present 
data, none of the genes proposed to link p53 and NER [growth arrest and DNA damage inducible alpha $(G A D D 45 A)$, proliferating cell nuclear antigen (PCNA) and apurinic/apyrimidinic endodeoxyribonuclease 1(APE1)] [26] were expressed. Moreover, GADD45GIP1, a gene induced by p53 and that inhibits G1 to $S$ phase of cell cycle progression was, in fact, down-regulated. Therefore, the present study suggests the possible role of alternative mechanisms for OSe in maintaining genomic stability in PEB through TC-NER. The present data suggests two likely possibilities, the activation of caspases by C-terminal-modified TXNR or ubiquitylation of damage-arrested RNA polymerase.

C-terminal-modified TXNR is unable to reduce TXN, but may replace the p53 apoptotic function via activation of caspases [27]. Interesting, Dalto et al. [3] reported a down-regulation of TXN in $\mathrm{OSeB}_{6} 10 \times \mathrm{CONT}$ and $\mathrm{OSeB}_{6} 10 \times \mathrm{MSeB}_{6} 10$, whereas in the present study $C A S P 3,6$, and 7 were up-regulated in these comparisons. For damage-arrested RNA polymerase, it might be released from the template by a mechanism that leads to its ubiquitylation and degradation [22], in which genes ERCC excision repair (ERCC) and cullin (CUL) play a main role. In $\mathrm{OSeB}_{6} 10 \times \mathrm{CONT}$, genes encoding proteins that are important in ubiquitination of proteins involved in cell cycle progression, signal transduction and transcription were up-regulated [ANAPC1, CUL1, CUL2, DDB1 and CUL4 associated factor 7 (DCAF7) and ERCC8]. Globally, these results on DNA repair are consistent with the numerically greater amount of viable embryos collected from $\mathrm{OSeB}_{6} 10$ gilts by Dalto et al. [3], and this, in absence of degenerated embryos.

\section{mRNA processing}

After the formation of the precursor mRNa (pre-mRNA) in the nucleus, it has to be converted to mature mRNA by splicing [28] in order to be translated. Pre-mRNA splicing occurs in the spliceosome, a large ribonucleoprotein complex composed of five U-type small nuclear ribonucleoprotein particles (snRNPs) and splicing factors [29].

The present data for $\mathrm{OSeB}_{6} 10 \times \mathrm{CONT}$ indicates that OSe acts on the ribonucleoprotein complex assembly. Although SeMet may have a structural function by replacing methionine in proteins, in PEB the most important effect of OSe appears to be on splicing factors activity. In fact, many dead-box proteins, pre-mRNA processing factors, snRNPs polypeptides, and other splicing factors (among them serine/arginine-rich splicing factors, poly (A) polymerases, and cleavage stimulation factors $3^{\prime}$ pre RNA) were impacted by maternal OSe supplementation.

During the second catalytic step of splicing, the exon junction complex (EJC) is formed on the pre-mRNA strand at the exon-exon junction [30,31]. In the present data for $\mathrm{OSeB}_{6} 10 \times \mathrm{CONT}$, two [eukaryotic translation initiation factor 4A3 (EIF4A3) and mago homolog, exon junction complex core component $(M A G O H)$ ] of the three core proteins of the EJC were expressed. This protein complex has major influences on translation, localization of the spliced mRNA, and mRNA surveillance. According to Brogna \& Wen [32], in mammalian cells, EJC mediates the link between splicing and nonsense-mediated decay pathway (NMD), a surveillance translation-coupled mechanism that eliminates mRNAs containing premature translationtermination codons [33].

The NMD pathway is assumed to affect selenoproteins expression because they contain multiple SeCys residues, which are encoded by the UGA codon that normally signals translation termination. In selenoproteins, however, SeCys insertion sequence (SECIS) recognizes UGA as a SeCys codon rather than as a stop signal. Under Se deficiency, Seyedali \& Berry [34] showed that NMD may act on selenoproteins mRNA. In the present data, SECIS binding protein 2 (SECISBP2) was up-regulated in $\mathrm{OSeB}_{6} 10 \times \mathrm{MSeB}_{6} 10$, suggesting that selenoproteins synthesis was active. In fact, selenoprotein $K$ was up-regulated in both $\mathrm{OSeB}_{6} 10 \times \mathrm{CONT}$ and $\mathrm{OSeB}_{6} 10 \mathrm{x}$ $\mathrm{MSeB}_{6} 10$. For the most known selenoproteins (glutathione peroxidases, iodothyronine deiodinases, and thioredoxin reductases), none of them was differentially expressed. This, however, does not imply that their synthesis was impaired but that maternal Se supplementation did not further stimulate their synthesis compared to the control diet or between Se sources.

\section{mRNA translation}

The present data indicates that besides the function of OSe as a structural component of the ribosome, both MSe and OSe play roles in translation, stimulating RNA binding factors and influencing translational initiation. A deeper evaluation of individual genes in these GO terms revealed that, under the present experimental conditions, Se is likely to influence specifically the Cap-dependent initiation. This process involves the interaction of the eukaryotic translation initiation factor (eIF) complex and the $5^{\prime}$ cap as well as with the $5^{\prime}$ untranslated region [35]. Additionally, the transport of the initiator tRNA, which encodes the amino acid methionine in eukaryotes, to the P-site of the small ribosomal subunit is performed by eIF2. This protein is also responsible for signaling the dissociation of several factors from the small ribosomal subunit, among them eIF3 that avoids the premature binding of the large ribosomal subunit, leading to the association of the large subunit and translation elongation [36]. Except for eIF5 and eIF6 that were not expressed by $\mathrm{MSeB}_{6} 10 \times \mathrm{CONT}$, all three comparisons have expressed many subunits of important eIFs. 


\section{Intracellular ceramides synthesis}

mRNA translation was expectedly related with peptide biosynthesis, which in turn was related with amide biosynthesis. Although the association between amides and peptides may be due to the presence of amide bonds in the forming protein, a deeper evaluation of individual genes revealed that the $\mathrm{GO}$ term amide biosynthetic process had many genes related with two out of the three pathways of ceramides biosynthesis.

The de novo pathway begins with the formation of 3-keto-dihydro-sphingosine from palmitoyl-CoA and serine by serine palmitoyl-transferase (SPTLC2) in the endoplasmic reticulum. Further, 3-keto-dihydro-sphingosine is reduced by 3-keto-dihydro-sphingosine reductase (KDSR) to form dihydro-sphingosine followed by an acylation by ceramide synthase (CERS1, CERS5, and CERS6) to form dihydro-ceramide, which is desaturated by delta 4-desaturase (DEGS) to form ceramide. Ceramide is subsequently transported to the Golgi apparatus where it is further metabolized and the outcomes transported to the plasma membrane [37].

Breakdown pathways allow the reversion of sphingolipids back to ceramide. One of the most biologically important reactions is the breakdown of sphingomyelin in the cell membrane releasing ceramide. Considering that sphingomyelin is the most abundant complex sphingolipid in human cells, its coordinated breakdown is an essential part of membrane homeostasis [38]. Besides the breakdown of sphingosine by ceramide synthases, the present results support that OSe plays a role in the breakdown of sphingomyelin by sphingomyelin phosphodiesterase (SMPD4 and SMPDL3A) and in the synthesis of sphingosine by $\mathrm{N}$-acylsphingosine amidohydrolase 1 (ASAH1) and/ or alkaline ceramidase 3 (ACER3). Sphingosine may enter the cell or it is metabolized by sphingosine kinase 1 (SPHK1), a protein regulated by SPHK1-interacting protein (SPHKAP), to form sphingosine-1-phosphate. The latter may be dephosphorylated by phospholipid phosphatase 1 (PPAP2A) to resynthesize sphingosine. In the cell, sphingosine can either go directly to the endoplasmic reticulum or enter the mitochondria where it will be metabolized back to ceramide and released into the endoplasmic reticulum [37]. Among all genes mentioned in de novo and breakdown pathways, only KDSR and SPHK1 were not expressed in $\mathrm{OSeB}_{6} 10 \times \mathrm{CONT}$ and/or $\mathrm{OSeB}_{6} 10 \times \mathrm{MSeB}_{6} 10$.

Simple sphingolipids have significant signaling and regulatory roles within cells, with serious consequences for mammalian physiology [37]. Ceramide and sphingosine-1phosphate have been shown to be important mediators in the signaling cascades involved in apoptosis, proliferation, differentiation, cell growth arrest, inflammation, cell migration and adhesion. In this sense, many factors known to promote the synthesis of sphingosine-1-phosphate [39] were expressed in the present data [platelet-derived growth factor subunit A (PDGFRA), insulin-like growth factor 1 receptor (IGF1R), vascular endothelial growth factor $\beta$ (VEGFB), tumor necrosis factor-related genes (TNFRSF21, TNFAIP8L3, TNFSF4, and TNFRSF9), and low-density lipoprotein receptors (LRP2 and LRP6). Globally, these results are once again consistent with the numerically greater amount of advanced-stage embryos collected from $\mathrm{OSeB}_{6} 10$ gilts by Dalto et al. [3].

\section{Intracellular peptides trafficking}

Besides the effects on peptides and lipid-related metabolites biosynthesis, $\mathrm{OSeB}_{6} 10 \times \mathrm{CONT}$ and $\mathrm{OSeB}_{6} 10 \times \mathrm{MSeB}_{6} 10$ comparisons also showed an impact on their transport via the signal recognition particle (SRP)-dependent cotranslational pathway of protein targeting to membrane. The cotranslational pathway uses SRP to deliver secretory proteins to a membrane-bound protein-conducting channel (translocon), which is present in the endoplasmic reticulum membrane, concomitantly with their synthesis in ribosomes [40]. In mammals, SRP consists of six proteins (encoded by SRP9, SRP14, SRP19, SRP54, SRP68 and SRP72) and a 7S RNA, most of which had genes expressed in the above mentioned comparisons. This process begins with the recognition of the signal peptide of the protein by SRP during the protein synthesis in the ribosome [41], with further insertion of the nascent protein into the translocon [42]. The translocon complex consists of oligosaccharyl transferase complex, the translocon-associated protein (TRAP) complex, and the translocating chain-associating membrane protein (TRAM), besides the central element Sec61 [43]. For the present data, many genes related with the translocon complex (3 SEC61 subunits, STT3A, STT3B, and 7 TRAPPC subunits) were expressed in $\mathrm{OSeB}_{6} 10 \times \mathrm{MSeB}_{6} 10$ and/or $\mathrm{OSeB}_{6} 10 \times \mathrm{CONT}$. Once the nascent polypeptide has been translocated into the endoplasmic reticulum membrane, the signal sequence is cleaved by signal peptidases (GTP hydrolysis) [44, 45], some of which were expressed (SPCS1, SPCS2, and SPCS3) in $\mathrm{OSeB}_{6} 10 \mathrm{x}$ $\mathrm{MSeB}_{6} 10$ and/or $\mathrm{OSeB}_{6} 10 \times \mathrm{CONT}$.

Signal peptidases are also found in the mitochondria import machinery [46]. The pre-protein containing peptide signals targeting the mitochondria is bound by translocases of outer membrane (TOM) and transported through the intermembrane space by translocases of inner membrane (TIM) [47]. Among the three mitochondrial TOMs, those responsible for binding pre-sequences and internal targeting peptides (TOM20 and TOM22) were expressed in $\mathrm{OSeB}_{6} 10 \times \mathrm{MSeB}_{6} 10$ and $\mathrm{OSeB}_{6} 10 \times \mathrm{CONT}$. Additionally, TIM23, which acts as a translocator of pre-proteins for the mitochondrial matrix, the inner membrane, and the intermembrane space, was affected in these same comparisons.

Exclusively for the comparison $\mathrm{OSeB}_{6} 10 \times \mathrm{CONT}$, the present data show an effect on mitochondrial respiratory 
chain complex assembly, more specifically the NADH dehydrogenase (ubiquinone) activity (21 NADH dehydrogenase and 5 cytochrome c genes stimulated). The evaluation of individual genes in these GO terms showed that Se acts mainly as a structural component, contrary to its expected antioxidant action against reactive oxygen species in the electron transport chain.

\section{Epigenetics}

It is already known that maternal diet may play a crucial role in epigenetic programming of conceptus development [48] and that methyl dietary supplements (such as SeMet) can alter the methylation of specific imprinted genes [49]. For this reason, an individual search for epigenetic-related genes was performed. Many important genes related with epigenetic events were expressed in $\mathrm{OSeB}_{6} 10 \quad \mathrm{x} \quad \mathrm{MSeB}_{6} 10$ and/or $\mathrm{OSeB}_{6} 10$ x CONT (DNMT1, DNMT3B and 19 other methyltransferases as well as MBD3, MBD4, MBD3L5, HAT1, HDAC1, $H D A C 9, K D M 5 C$, and WDR5) whereas only a few in $\mathrm{MSeB}_{6} 10 \times \mathrm{CONT}$ (HDAC1, HDAC9, KDM5C, and 6 methyltransferases).

Epigenetic processes are dynamic during embryogenesis partially because of the significant amount of DNA synthesis that occurs in this period [50]. During the pre-implantation period, embryos' epigenome is particularly susceptible to environmentally induced modifications. At this period, de/methylation of DNA and histones modification occurs. Effects of supplemental Se on global and gene-specific DNA methylation have been reported [51, 52], but never in PEB after maternal Se supplementation. Transfer of methyl groups from SAM to the 5-carbon position of cytosine by DNA methyltransferases (DNMTs), results in 5-methylcytosine (5 $\mathrm{mC}$ ) [53]. In contrast, DNA demethylation is not catalyzed directly but results from either DNA replication-coupled dilution or replacement of $5 \mathrm{mC}$. For histones, the interference of nutrients occurs mainly through modulation of histone modifying enzymes and via interference with substrate availability. According to Narayan et al. [54], selenoprotein biosynthesis is crucial for selenite-induced modulation of histone $\mathrm{H} 4$ acetylation, supporting the conclusion of Dalto et al. [3] that, although not influenced by Se sources with $B_{6}, P E B$ are potentially capable of synthesizing selenoproteins.

A tempting interpretation for the higher impact of OSe (SeMet) compared to MSe (selenite) on epigenetic events would be to link it to the synthesis of SAM during the demethylation of SeMet to Se-homocysteine. However, as the metabolic ratio between methionine and SeMet is generally considered to be above 3000/1 [55], it appears likely that the contribution of SeMet to SAM (a sulfurized metabolite) synthesis is negligible. Nevertheless, this is dependent upon the specific biosynthetic substitution of methionine by SeMet in PEB cells which has never been determined in pigs. Using recombinant human annexin $\mathrm{V}$ expressed in $E$. coli as a model, Budisa et al. [56] showed that, independently of experimental SeMet concentration (ranged from 0.3 to $0.8 \mathrm{mM}$ ), methionine was fully replace by SeMet. However, it has to be stated that the biosynthetic substitution of methionine by SeMet may vary considerably between species.

If the above mentioned ratio of 3000/1 between methionine and SeMet also applies to PEB, it has to be assumed that it is reflected on intermediary metabolites of the transmethylation pathway, such as Se-homocysteine. Therefore, the demethylation of SeMet to Se-homocysteine would also be quantitatively negligible within the total (sulfur $+\mathrm{Se}$ ) homocysteine levels. However, some studies showed an inverse correlation between Se status and total (sulfur $+\mathrm{Se}$ ) homocysteine levels $[57,58]$ indicating the important impact of Se on intermediary metabolites of the transmethylation pathway. Consequently, the question remains as whether selenized molecules would be more active than sulfurized ones within the transmethylation pathway or whether alternative Se-dependent metabolic pathways would be present in PEB to influence epigenetic events.

\section{Conclusions}

Global gene expression of PEB from OSe supplemented gilts was more impacted than those of MSe supplemented gilts. Maternal OSe supplementation influenced PEB genomic stability, intracellular ceramides synthesis and peptides trafficking, whereas maternal MSe supplementation was restricted to RNA binding. Different concentrations of Se metabolites (SeMet and SeCys) in PEB could explain these great differences based on their distinctive metabolic pathways and functions. Although the present study allows only inferences in this regard, these results are consistent with the better morphological and physiological development of embryos from $\mathrm{OSeB}_{6} 10$ supplemented gilts as previously reported by this laboratory [3].

Considering the particular transfer of Se from gilts to PEB, the immature transsulfuration pathway generating two metabolic pathways for Se (one for SeMet and other for SeCys), and the possible existence of alternative pathways for epigenetic control, the impact of maternal Se supplementation on transmethylation and transsulfuration pathways in PEB deserves to be further explored. 


\section{Additional files}

Additional file 1: Table S1. List of unique genes symbols from reannotated EMPV1 used as background for Gene Ontology Enrichment Analysis. (XLSX $817 \mathrm{~kb}$ )

Additional file 2: Table S2. Complete list of expressed genes related with $\mathrm{MSeB}_{6} 10$ vs CONT, OSeB 610 vs CONT, and $\mathrm{OSeB}_{6} 10$ vs MSeB 610 comparisons. (XLSX $508 \mathrm{~kb}$ )

Additional file 3: Table S3. Description of data: Complete list of Gene Ontology terms (GORILLA and REVIGO) related with $\mathrm{MSeB}_{6} 10$ vs CONT, $\mathrm{OSeB}_{6} 10$ vs CONT, and $\mathrm{OSeB}_{6} 10$ vs MSeB 610 comparisons. (XLSX 1678 kb)

\section{Abbreviations}

$\mathrm{B}_{6}$ : Vitamin $\mathrm{B}_{6}$; CONT: Control treatment; EJC: Exon junction complex; GO: Gene ontology; GS: Gene symbol; MSe: Mineral selenium; MSeB 6 10: Basa diet +0.3 and $10 \mathrm{mg} / \mathrm{kg}$ of supplemental inorganic Se and $\mathrm{HCl}$-pyridoxine; NER: Nucleotide excision repair; NMD: Nonsense-mediated decay; OSe: Organic selenium; OSeB 6 10: Basal diet +0.3 and $10 \mathrm{mg} / \mathrm{kg}$ of supplemental organic Se and HCl-pyridoxine; PEB: Porcine expanded blastocyst; pre-mRNA: Precursor mRNa; RT-qPCR: Quantitative real-time RTPCR; SAM: S-adenosyl methionine; Se: Selenium; SECIS: Selenocysteine insertion sequence; SeCys: Selenocysteine; Se-GPX: Selenium-dependent glutathione peroxidase; SeMet: Selenomethionine; snRNP: Small nuclear ribonucleoprotein particle; SRP: Signal recognition particle; TCNER: Transcription-coupled nucleotide excision repair; TIM: Translocases of inner membrane; TOM: Translocases of outer membrane;

TRAM: Translocating chain-associating membrane protein; TRAP: Transloconassociated protein

\section{Acknowledgements}

Not applicable.

\section{Funding}

This research did not receive any specific grant from any funding agency in the public, commercial or not-for-profit sector.

\section{Availability of data and materials}

All data generated or analyzed during this study are included in this published article or its supplementary information files.

\section{Authors' contributions}

DBD analyzed all data, performed statistical analysis, interpreted results and wrote the manuscript. ST revised the dataset and the statistical analysis. MKD and J-JM revised technical and scientific aspects of the manuscript, and were contributors in writing the manuscript. All authors read and approved the final manuscript

\section{Ethics approval}

Experimental procedures were carried out following the guidelines of the Canadian Council on Animal Care (2009) and were approved by the Institutional Animal Care Committee of the Sherbrooke Research and Development Centre, Quebec, Canada (\#400). All animals were cared for and slaughtered according to the recommended code of practice of Agriculture Canada (1993)

\section{Consent for publication}

Not applicable.

\section{Competing interests}

The authors declare that they have no competing interests.

\section{Publisher's Note}

Springer Nature remains neutral with regard to jurisdictional claims in published maps and institutional affiliations.

\section{Author details}

'Sherbrooke Research and Development Centre, Agriculture and Agri-Food Canada, 2000 College Street, Sherbrooke, QC J1M 0C8, Canada. ²Department of Agricultural, Food and Nutritional Science, University of Alberta, Edmonton, AB T6G 2P5, Canada.
Received: 18 October 2017 Accepted: 9 November 2018

Published online: 21 November 2018

\section{References}

1. Gardiner CS, Reed DJ. Glutathione redox cycle-driven recovery of reduced glutathione after oxidation by tertiary-butyl hydroperoxide in preimplantation mouse embryos. Arch Biochem Biophys. 1995;321:6-12. https://doi.org/10.1006/abbi.1995.1361.

2. Levonen AL, Lapatto R, Saksela M, Raivio KO. Human cystathioninegammalyase: developmental and in vitro expression of two isoforms. Biochem J. 2000:347:291-5. https://doi.org/10.1042/bj3470291.

3. Dalto DB, Tsoi S, Audet I, Dyck MK, Foxcroft GR, Matte JJ. Gene expression of porcine blastocysts from gilts fed organic or inorganic selenium and pyridoxine. Reproduction. 2015;149:31-42. https://doi.org/ 10.1530/REP-14-0408.

4. Schrauzer GN. The nutritional significance, metabolism and toxicology of selenomethionine. Adv Food Nutr Res. 2003:47:73-112. https://doi.org/10. 1016/S1043-4526(03)47002-2

5. Dalto DB, Matte JJ. Pyridoxine (vitamin $B_{6}$ ) and the glutathione peroxidase system; a link between one-carbon metabolism and antioxidation. Nutrients. 2017;9:189-202. https://doi.org/10.3390/nu9030189.

6. Joseph J, Loscalzo J. Methoxistasis: integrating the roles of homocysteine and folic acid in cardiovascular pathobiology. Nutrients. 2013;5:3235-56. https://doi.org/10.3390/nu5083235.

7. Kalhan SC. One carbon metabolism in pregnancy: impact on maternal, fetal and neonatal health. Mol Cell Endocrinol. 2016:435:48-60. https://doi.org/10. 1016/j.mce.2016.06.006.

8. Selhub J. Homocysteine metabolism. Annu Rev Nutr. 1999;19:217-46.

9. Berg JM, Tymoczko JL, Stryer L. Biochemistry. 5th ed. New York: WH Freeman and Company; 2002.

10. D'Aquila P, Bellizzi D, Passarino G. Mitochondria in health, aging and diseases: the epigenetic perspective. Biogerontology. 2015;16:569-85. https://doi.org/10.1007/s10522-015-9562-3.

11. NRC. Nutrient Requirements of Swine. 11th ed. Washington: National Academic Press; 2012

12. Konig R, Baldessari D, Pollet N, Niehrs C, Eils R. Reliability of gene expression ratios for CDNA microarrays in multiconditional experiments with a reference design. Nucleic Acids Res. 2004;32:e29. https://doi.org/10.1093/ nar/gnh027.

13. Pfaffl MW, Horgan GW, Dempfle L. Relative expression software tool (REST) for group-wise comparison and statistical analysis of relative expression results in real-time PCR. Nucleic Acids Res. 2002;30:e36. https://doi.org/10. 1093/nar/30.9.e36.

14. Burk RF, Olson GE, Hill KE, Winfrey VP, Motley AK, Kurokawa S. Maternal-fetal transfer of selenium in the mouse. FASEB J. 2013;27:3249-56. https://doi. org/10.1096/fj.13-231852

15. Fortier ME, Audet I, Giguère A, Laforest JP, Bilodeau JF, Quesnel H, Matte JJ. Effect of dietary organic and inorganic selenium on antioxidant status, embryo development, and reproductive performance in hyperovulatory first-parity gilts. J Anim Sci. 2012:90:231-40. https://doi.org/10.2527/jas.2010-3340.

16. Dalto DB, Audet I, Lapointe J, Matte JJ. The importance of pyridoxine for the impact of the dietary selenium sources on redox balance, embryo development, and reproductive performance in gilts. J Trace Elem Med Biol. 2016;34:79-89. https://doi.org/10.1016/j.jtemb.2016.01.001.

17. Rouse J, Jackson SP. Interfaces between the detection, signaling, and repair of DNA damage. Science. 2002;297:547-51. https://doi.org/10.1126/science. 1074740.

18. Ljungman M, Lane DP. Transcription-guarding the genome by sensing DNA damage. Nat Rev Cancer. 2004;4:727-37. https://doi.org/10.1038/nrc1435.

19. Bohr VA, Smith CA, Okumoto DS, Hanawalt PC. DNA repair in an active gene: removal of pyrimidine dimers from the DHFR gene of $\mathrm{CHO}$ cells is much more efficient than in the genome overall. Cell. 1985;40:359-69.

20. Mellon I, Spivak G, Hanawalt PC. Selective removal of transcription- blocking DNA damage from the transcribed strand of the mammalian DHFR gene. Cell. 1987;51:241-9.

21. Seo YR, Kelley MR, Smith ML. Selenomethionine regulation of p53 by a ref1dependent redox mechanism. P Natl Acad Sci USA. 2002;99:14548-53. https://doi.org/10.1073/pnas.212319799.

22. Fousteri M, Mullenders LHF. Transcription-coupled nucleotide excision repair in mammalian cells: molecular mechanisms and biological effects. Cell Res. 2008;18:73-84. https://doi.org/10.1038/cr.2008.6. 
23. Offer H, Zurer I, Banfalvi G, Reha'k M, Falcovitz A, Milyavsky M, et al. p53 modulates base excision repair activity in a cell cycle-specific manner after genotoxic stress. Cancer Res. 2001;61:88-96.

24. Zhou J, Ahn J, Wilson SH, Prives C. A role for p53 in base excision repair. EMBO J. 2001;20:914-23. https://doi.org/10.1093/emboj/20.4.914.

25. Lee YS, Chung MH. Base excision repair synthesis of DNA containing 8oxoguanine in Escherichia coli. Exp Mol Med. 2003;35:106-12. https://doi. org/10.1038/emm.2003.15.

26. Jung HJ, Kim HL, Kim YJ, Weon J, Seo YR. A novel chemopreventive mechanism of selenomethionine: enhancement of APE1 enzyme activity via a Gadd45a, PCNA and APE1 protein complex that regulates p53-mediated base excision repair. Oncol Rep. 2013;30:1581-6. https://doi.org/10.3892/or 2013.2613.

27. Cassidy PB, Edes K, Nelson CC, Parsawar K, Fitzpatrick FA, Moos PJ. Thioredoxin reductase is required for the inactivation of tumor suppressor p53 and for apoptosis induced by endogenous electrophiles. Carcinogenesis. 2006;27:2538-49.

28. Berget SM, Moore C, Sharp PA. Spliced segments at the 50 terminus of adenovirus 2 late mRNA. P Natl Acad Sci USA. 1977;74:3171-5.

29. Valadkhan S, Jaladat $Y$. The spliceosomal proteome: at the heart of the largest cellular ribonucleoprotein machine. Proteomics. 2010;10:4128-41. https://doi.org/10.1002/pmic.201000354.

30. Le Hir H, Izaurralde E, Maquat LE, Moore MJ. The spliceosome deposits multiple proteins 20-24 nucleotides upstream of mRNA exon-exon junctions. EMBO J. 2000;19:6860-9. https://doi.org/10.1093/emboj/19.24.6860.

31. Reichert VL, Le Hir H, Jurica MS, Moore MJ. 5' exon interactions within the spliceosome establish a framework for exon junction complex structure and assembly. Genes Dev. 2002;16:2778-91. https://doi.org/10.1101/gad.1030602.

32. Brogna S, Wen J. Nonsense-mediated mRNA decay (NMD) mechanisms. Nat Struct Mol Biol. 2009;16:107-13. https://doi.org/10.1038/nsmb.1550.

33. He F, Peltz SW, Donahue JL, Rosbash M, Jacobson A. Stabilization and ribosome association of unspliced pre-mRNAs in a yeast Upf1- mutant. P Natl Acad Sci USA. 1993;90:7034-8.

34. Seyedali A, Berry MJ. Nonsense-mediated decay factors are involved in the regulation of selenoprotein mRNA levels during selenium deficiency. RNA. 2014;20:1248-56. https://doi.org/10.1261/rna.043463.113.

35. Hellen CU, Sarnow P. Internal ribosome entry sites in eukaryotic mRNA molecules. Genes Dev. 2001;15:1593-612. https://doi.org/10.1101/gad.891101.

36. Malys N, McCarthy JEG. Translation initiation: variations in the mechanism can be anticipated. Cell Mol Life Sci. 2011;68:991-1003. https://doi.org/10. 1007/s00018-010-0588-z.

37. Gault CR, Obeid LM, Hannun YA. An overview of sphingolipid metabolism: from synthesis to breakdown. Adv Exp Med Biol. 2010;688:1-23.

38. Green DR. Apoptosis and sphingomyelin hydrolysis: the flip side. J Cell Biol. 2000;150:F5-7.

39. Hannun YA, Obeid LM. Principles of bioactive lipid signalling: lessons from sphingolipids. Nat Rev Mol Cell Biol. 2008;9:139-50. https://doi.org/10.1038/ nrm2329.

40. Keenan RJ, Freymann DM, Stroud RM, Walter P. The signal recognition particle. Annu Rev Biochem. 2001;70:755-75. https://doi.org/10.1146/ annurev.biochem.70.1.755.

41. Nyathi Y, Wilkinson BM, Pool MR. Co-translational targeting and translocation of proteins to the endoplasmic reticulum. Biochim Biophys Acta. 1833;2013:2392-402. https://doi.org/10.1016/j.bbamcr.2013.02.021.

42. Rapoport T. Protein translocation across the eukaryotic endoplasmic reticulum and bacterial plasma membranes. Nature. 2007;450:663-9. https://doi.org/10. 1038/nature06384

43. Johnson $A E$, van Waes MA. The translocon: a dynamic gateway at the ER membrane. Annu Rev Cell Dev Biol. 1999;15:799-842. https://doi.org/10. 1146/annurev.cellbio.15.1.799.

44. Miller JD, Wilhelm H, Gierasch L, Gilmore R, Walter P. GTP binding and hydrolysis by the signal recognition particle during initiation of protein translocation. Nature. 1993;366:351-4. https://doi.org/10.1038/366351a0.

45. Grudnik $P$, Bange $G$, Sinning I. Protein targeting by the signal recognition particle. Biol Chem. 2009;390:775-82. https://doi.org/10.1515/BC.2009.102.

46. Paetzel M, Karla A, Strynadka NC, Dalbey RE. Signal peptidases. Chem Rev. 2002;102:4549-80. https://doi.org/10.1021/cr010166y.

47. Bolender N, Sickmann A, Wagner R, Meisinger C, Pfanner N. Multiple pathways for sorting mitochondrial precursor proteins. EMBO Rep. 2008;9:42-9. https://doi.org/10.1038/sj.embor.7401126.
48. van Engeland $M$, Weijenberg MP, Roemen GM, Brink M, de Bruine AP, Goldbohm RA, et al. Effects of dietary folate and alcohol intake on promote methylation in sporadic colorectal cancer: the Netherlands cohort study on diet and cancer. Cancer Res. 2003;63:3133-7.

49. Cooney CA, Dave AA, Wolff GL. Maternal methyl supplements in mice affect epigenetic variation and DNA methylation of offspring. J Nutr. 2002;132: 2393S-400S.

50. Smith ZD, Chan MM, Humm KC, Karnik R, Mekhoubad S, Regev A, et al. DNA methylation dynamics of the human preimplantation embryo. Nature. 2014;511:611-5. https://doi.org/10.1038/nature13581.

51. Davis CD, Uthus EO, Finley JW. Dietary selenium and arsenic affect DNA methylation in vitro in Caco-2 cells and in vivo in rat liver and colon. J Nutr. 2000;130:2903-9.

52. Zeng $H$, Yan $L$, Cheng WH, Uthus EO. Dietary selenomethionine increases exon-specific DNA methylation of the p53 gene in rat liver and colon mucosa. J Nutr. 2011;141:1464-8. https://doi.org/10.3945/jn.111.140715.

53. Speckmann B, Grune T. Epigenetic effects of selenium and their implications for health. Epigenetics. 2015;10:179-90. https://doi.org/10.1080/15592294. 2015.1013792

54. Narayan V, Ravindra KC, Liao C, Kaushal N, Carlson BA, Prabhu KS. Epigenetic regulation of inflammatory gene expression in macrophages by selenium. J Nutr Biochem. 2014:26:138-45. https://doi.org/10.1016/j.jnutbio.2014.09.009.

55. Rýdlová M, Růnová K, Száková J, Fučíková A, Hakenová A, Mlejnek P, et al. The Response of Macro- and Micronutrient Nutrient Status and Biochemical Processes in Rats Fed on a Diet with Selenium-Enriched Defatted Rapeseed and/or Vitamin E Supplementation. Biomed Res Int. 2017:art6759810. https://doi.org/10.1155/2017/6759810.

56. Budisa N, Steipe B, Demange P, Eckerskorn C, Kellermann J, Huber R. Highlevel biosynthetic substitution of methionine in proteins by its analogs 2aminohexanoic acid, selenomethionine, telluromethionine and ethionine in Escherichia coli. Eur J Biochem. 1995;230:788-96. https://doi.org/10.1111/j. 1432-1033.1995.0788h.x.

57. Belanger MC, Dewailly E, Berthiaume L, Noel M, Bergeron J, Mirault ME, Julien P. Dietary contaminants and oxidative stress in Inuit of Nunavik. Metabolism. 2006:55:989-95. https://doi.org/10.1016/j.metabol.2006.03.007.

58. Bekaert B, Cooper ML, Green FR, McNulty H, Pentieva K, Scott JM, et al. Effect of selenium status and supplementation with high-selenium yeast on plasma homocysteine and B vitamin concentrations in the UK elderly. Mo Nutr Food Res. 2008:52:1324-33. https://doi.org/10.1002/mnfr.200700353.

Ready to submit your research? Choose BMC and benefit from:

- fast, convenient online submission

- thorough peer review by experienced researchers in your field

- rapid publication on acceptance

- support for research data, including large and complex data types

- gold Open Access which fosters wider collaboration and increased citations

- maximum visibility for your research: over $100 \mathrm{M}$ website views per year

At $\mathrm{BMC}$, research is always in progress.

Learn more biomedcentral.com/submissions 